\title{
Does a medical history of hypertension influence disclosing genetic testing results of the risk for salt-sensitive hypertension, in primary care?
}

\author{
This article was published in the following Dove Press journal: \\ International Journal of General Medicine \\ 27 July 2016 \\ Number of times this article has been viewed
}

\author{
Masanobu Okayama ${ }^{1,2}$ \\ Taro Takeshima ${ }^{2}$ \\ Masanori Harada ${ }^{3}$ \\ Ryusuke $\mathrm{Ae}^{4}$ \\ Eiji Kajii ${ }^{2}$
}

'Division of Community Medicine and Medical Education, Kobe University Graduate School of Medicine, Kobe, Hyogo, ${ }^{2}$ Division of Community and Family Medicine, Center for Community Medicine, Jichi Medical University, Shimotsuke, Tochigi, ${ }^{3}$ Department of Support of Rural Medicine, Yamaguchi Grand Medical Center, Hofu, Yamaguchi, ${ }^{4}$ Division of Public Health, Center for Community Medicine, Jichi Medical University, Shimotsuke, Tochigi, Japan
Correspondence: Masanobu Okayama Division of Community Medicine and Medical Education, Kobe University Graduate School of Medicine, Arata-cho 2-I-5, Hyogo-ku, Kobe, Hyogo 652-0032, Japan

Tel +8I 783826732

Fax +8I 783826283

Email okayama@jichi.ac.jp
Objective: Disclosing genetic testing results may contribute to the prevention and management of many common diseases. However, whether the presence of a disease influences these effects is unclear. This study aimed to clarify the difference in the effects of disclosing genetic testing results of the risk for developing salt-sensitive hypertension on the behavioral modifications with respect to salt intake in hypertensive and nonhypertensive patients.

Methods: A cross-sectional study using a self-administered questionnaire was conducted for outpatients aged $>20$ years $(\mathrm{N}=2,237)$ at six primary care clinics and hospitals in Japan. The main factors assessed were medical histories of hypertension, salt preferences, reduced salt intakes, and behavior modifications for reducing salt intake. Behavioral modifications of participants were assessed using their behavior stages before and after disclosure of the hypothetical genetic testing results.

Results: Of the 2,237 participants, 1,644 (73.5\%) responded to the survey. Of these respondents, $558(33.9 \%)$ patients were hypertensive and 1,086 (66.1\%) were nonhypertensive. After being notified of the result "If with genetic risk", the nonhypertensive participants were more likely to make positive behavioral modifications compared to the hypertensive patients among all participants and in those aged $<65$ years (adjusted relative ratio [ad-RR], 1.76; 95\% confidence interval, $1.12-2.76$ and ad-RR, $1.99 ; 1.11-3.57$, respectively). In contrast, no difference in negative behavioral modifications between hypertensive and nonhypertensive patients was detected after being notified of the result "If without genetic risk" (ad-RR, 1.05; 95\% confidence interval, 0.70-1.57). Conclusion: The behavior of modifying salt intake after disclosure of the genetic testing results differed between hypertensive and nonhypertensive patients. Disclosing a genetic risk for salt-sensitive hypertension was likely to cause nonhypertensive patients, especially those aged $<65$ years, to improve their behavior regarding salt intake. We conclude that disclosing genetic testing results could help prevent hypertension, and that the doctor should communicate the genetic testing results to those patients with a medical history of hypertension, or those who are at risk of developing hypertension.

Keywords: attitude to health, genetic testing, hypertension, outpatient, sodium-restricted

\section{Introduction}

A variety of factors affect the development of essential hypertension, and although the mechanism of onset is generally poorly understood, genetic factors have been implicated in the pathogenesis of this disorder. ${ }^{1,2}$ A study using ambulatory blood pressure monitoring suggested that genetic factors account for $30 \%-60 \%$ of the variation in blood 
pressure, ${ }^{3}$ whereas a variety of other risk factors, including excess sodium intake, excess alcohol intake, obesity, weight gain, and physical inactivity, have also been associated with essential hypertension, ${ }^{2}$ suggesting that an interaction between genetic and environmental factors is responsible for the development of hypertension. ${ }^{2}$

During the past few years, rapid advances in genetic research, especially genome-wide association analyses, have been responsible for detecting hundreds of single nucleotide polymorphisms (SNPs) significantly associated with common diseases such as hypertension, diabetes mellitus, and hyperlipidemia. ${ }^{4,5}$ These findings form the basis of personalized medicine, which has the potential to enhance human health through more effective prevention, diagnosis, and treatment. ${ }^{6}$ However, the vast majority of these SNPs are associated with very low odds ratios for common diseases. ${ }^{7,8}$

There is a real need for a breakthrough concerning the utility of the results of genetic research in clinical practice. Several studies have explored the psychological, behavioral, and clinical effects of disclosing the genetic risk for common diseases, although the findings have been mixed. ${ }^{9-17}$ Patients expect genetic testing to precisely predict whether they are at risk of developing a specific disease and thereby to improve their ability to manage this disease, but for patients already affected by common diseases, the notification of the genetic risk for these diseases may provide little or no effect on their behavior toward preventing the disease. ${ }^{9}{ }^{18}$ However, these previous studies have not given in-depth consideration to the influence of the patients' medical histories on the effect of disclosing a genetic risk. This oversight may be the cause of the inconsistent findings among the previous studies, and thus, solving this issue is essential to accurately determine the effects of genetic risk notification.

Reducing salt intake is an essential behavioral intervention for the prevention and treatment of hypertension. ${ }^{19,20}$ Furthermore, several gene polymorphisms associated with salt-sensitive hypertension have been identified, ${ }^{21-23}$ and the issue of whether disclosure of a genetic risk for salt-sensitive hypertension modifies the patient behavior regarding salt intake must be established in order to develop effective personalized medicine. Therefore, it is necessary to examine the influence of the patients' medical histories of hypertension on the effect of disclosing the genetic risk. The purpose of this study was to clarify the difference in the effects of disclosing genetic testing results of developing salt-sensitive hypertension on the behavioral modifications with respect to salt intake in hypertensive and nonhypertensive patients.

\section{Materials and methods \\ Study design}

This cross-sectional study used a self-administered questionnaire.

\section{Participants and measurements}

Anonymous questionnaires were distributed to outpatients aged $>20$ years who visited primary care departments in four clinics and two small hospitals in rural and suburban areas in Japan. Data were collected during a 2-week period at each clinic or hospital from September 2009 to February 2010. Patients were handed questionnaires at the reception desks, and were told that they would not be remunerated for participation and could decline to participate without penalty. The patients filled out the questionnaire in the waiting room. Primary care physicians were not informed as to whether or not the patients answered the questionnaires.

The questionnaire included questions about the age, sex, education level, occupation ("Are you a health care worker?" [yes/no]), family, personal medical history (with regard to hypertension, diabetes mellitus, stroke, and myocardial infarction), body mass index (BMI), worries about hypertension and diabetes mellitus ("Do you worry about hypertension?" and "Do you worry about diabetes mellitus?" [yes/relatively yes/relatively no/no]), salt preference ("Do you prefer salty foods?" [yes/no]), current lifestyle behaviors (cigarette smoking, alcohol consumption, regular exercise, and salt intake), behavior stage of reducing salt intake, willingness to undergo genetic testing for salt-sensitive hypertension, and the behavioral intention of reducing salt intake after notification of the genetic testing results. The behavior stage was selected from five questions: 1) "I am not concerned about reducing my salt intake at all" (no intention of salt restriction); 2) "I must reduce my salt intake, but I cannot do it" (have the intention but not prepared for salt restriction); 3 ) "I am ready to start reducing my salt intake" (prepared for salt restriction in the near future); 4) "I have already reduced my salt intake within the past 6 months" (successfully altering a behavior for 1 day to 6 months); and 5) "I have been reducing my salt intake for more than 6 months" (successfully altering a behavior for more than 6 months). These questions were adapted from a questionnaire based on the transtheoretical model of behavioral change authorized by the Ministry of Health, Labor and Welfare in Japan..$^{24}$

A description of genetic testing for salt-sensitive hypertension was provided using the following sentence: "The genetic testing detects whether or not you have a genetic risk predisposing you to hypertension by excessive salt intake", 
but no additional information was given. The willingness of the patients to be tested was determined by asking: "Would you want to undergo genetic testing for salt-sensitive hypertension?" (yes/no/do not know). The behavior intentions were measured as, the behavior stages of reducing salt intake examined after the patients had been notified of hypothetical positive or negative results of genetic testing for salt-sensitive hypertension ("If you are notified that you have a genetic risk for salt-sensitive hypertension" [If with genetic risk] and "If you are notified that you do not have a genetic risk for salt-sensitive hypertension" [If without genetic risk]). The behavior stage after notification was classified as one of the six stages, using the five questions mentioned earlier and one additional statement: "I will quit reducing salt intake" (quit salt restriction) (yes/no).

\section{Statistical analysis}

Statistical analyses were performed using the STATA/MP version 13.1 software (Stata Corp LP, College Station, TX, USA). A $P$-value $<0.05$ was considered significant. Before performing the descriptive analysis, participant age was divided into three categories $(<50,50-64$, and $\geq 65$ years old $)$. Obesity was defined as having a BMI $>25 \mathrm{~kg} / \mathrm{m}^{2} .{ }^{25}$ On the basis of the transtheoretical model, ${ }^{26}$ behavior stages were classified into six stages: precontemplation (no intention of salt restriction); contemplation (have the intention but not prepared for salt restriction); preparation (prepared for salt restriction in the near future); action (successfully altering the behavior for 1 day to 6 months); maintenance (successfully altering the behavior for more than 6 months); and relapse (quit salt restriction). We defined participants with a personal medical history of hypertension as hypertensive patients and those without hypertension as nonhypertensive patients. Descriptive statistics were reported as mean \pm standard deviation (SD) for age and as a proportion for all other variables and categories. The Student's $t$-test and chi-square test were used to compare the age and the proportion of participants between the hypertensive and nonhypertensive groups, respectively.

Behavioral modifications of participants were assessed using their behavior stages before and after being notified of hypothetical genetic testing results. The assessments of the behavioral modifications were performed by dividing the participants into two groups based on their current behavior regarding salt intake. Among participants who had not begun to reduce their salt intake, their behavioral modifications were divided into three categories: progress, no change, and regress, with an increase in $\geq 1$ stage after notification of the genetic testing results being defined as progress. For example, participants originally at the precontemplation stage, defined as being in the contemplation, preparation, or action stage following the notification, were classified as belonging to the "progress" category. On the other hand, participants showing a reduction in $\geq 1$ stage after the notification of the genetic testing results were classified as belonging to "regress" category, and participants without any change in behavior stages before and after the notification were classified as a "no change" group. Participants who had already reduced salt intake before the notification were divided into no change and relapse groups based on their current behavior stage.

To clarify the influences of medical history of hypertension on the behavioral changes after disclosing genetic test results, logistic regression analyses were conducted among all participants and subgroups to examine differences in proportions of behavioral modification following notification of hypothetical genetic test results between the hypertensive and nonhypertensive patient groups (those $<65$ years old and those $\geq 65$ years old). The progress in the participants' behavior stages, as a result of the notification, was classified as positive behavioral modifications and the regress or relapse as negative behavioral modifications.

Univariate logistic regression analysis was used to calculate the crude relative ratios (RRs) and 95\% confidence intervals (CIs). Adjusted RRs (95\% CIs), adjusted for age, sex, education status, salty food preferences, behavior stages before being notified of the genetic results, and willingness to be tested, were analyzed using multivariate logistic regression analysis based on the findings in our previous study. ${ }^{13}$ However, variables that predicted failure perfectly were not adopted in the statistical analyses, that is, the behavior stages of the patients before genetic information was disclosed were not considered for the analyses of the negative effect.

\section{Ethical statement}

Approval for the study and questionnaire was obtained from the Institutional Review Board of Jichi Medical University. Informed consent was obtained from each participant by responding to the questionnaire.

\section{Results}

Of the 2,237 outpatients who visited the study sites during the study period, 1,644 ( $73.5 \%$ response rate) completed the survey (581 males, 35.3\%; 1,063 females, 64.7\%). The mean age of the respondents was $57.3 \pm 17.6$ years, and the numbers of hypertensive patients and nonhypertensive patients were $558(33.9 \%)$ and $1,086(66.1 \%)$, respectively. The mean age of participants significantly differed between the hypertensive (69.5 \pm 12.4 years) and the nonhypertensive groups 
(50.9 \pm 16.5 years; $P<0.001, t$-test). Similarly, the proportion of all other items, except sex, family medical history of diabetes mellitus, and salt preference, differed significantly between the groups $(P<0.05$; Table 1$)$.

For participants who had not begun to reduce their salt intake prior to the notification, both the proportion of behavior stages and behavioral modifications after being notified of the result "If with genetic risk" differed between the hypertensive and nonhypertensive patient groups (Table 2). In contrast, if participants were notified of the result "If without genetic risk", there were no significant differences between the two groups. For participants who had already been reducing salt intake, no difference in the proportions of behavior stages or behavioral modifications

Table I Demographic characteristics of study participants

\begin{tabular}{|c|c|c|c|c|}
\hline Characteristics & $\begin{array}{l}\text { Total } \\
n=1,644, n \text { (\%) }\end{array}$ & $\begin{array}{l}\text { Hypertension } \\
n=558, n(\%)\end{array}$ & $\begin{array}{l}\text { Nonhypertension } \\
n=1,086, n(\%)\end{array}$ & $P$-value* \\
\hline Age (years) & & & & $<0.001$ \\
\hline$<50$ & $579(35.2)$ & $40(7.2)$ & $539(49.6)$ & \\
\hline $50-64$ & $426(25.9)$ & $140(25.1)$ & $286(26.3)$ & \\
\hline$\geq 65$ & $639(38.9)$ & $378(67.7)$ & $261(24.0)$ & \\
\hline Sex & & & & 0.107 \\
\hline Male & $58 \mathrm{I}(35.3)$ & $212(38.0)$ & $369(34.0)$ & \\
\hline Female & $1063(64.7)$ & $346(62.0)$ & $717(66.0)$ & \\
\hline Occupation & & & & $<0.001$ \\
\hline Health care worker & I I8 (7.2) & $16(2.9)$ & $102(9.4)$ & \\
\hline Not a health care worker & $1526(92.8)$ & $542(97.1)$ & $984(90.6)$ & \\
\hline Education level & & & & $<0.001$ \\
\hline Elementary school & $101(6.1)$ & $67(12.0)$ & $34(3.1)$ & \\
\hline Junior high school & $394(24.0)$ & $195(34.9)$ & $199(18.3)$ & \\
\hline High school & $620(37.7)$ & $197(35.3)$ & $423(39.0)$ & \\
\hline College & $362(22.0)$ & $64(I I .5)$ & $298(27.4)$ & \\
\hline University & $167(10.2)$ & $35(6.3)$ & $132(12.2)$ & \\
\hline \multicolumn{5}{|l|}{ Family medical history } \\
\hline Hypertension & 639 (38.9) & $284(50.9)$ & $355(32.7)$ & $<0.001$ \\
\hline Diabetes mellitus & $234(14.2)$ & $68(12.2)$ & $166(15.3)$ & 0.089 \\
\hline Stroke & $286(17.4)$ & $140(25.1)$ & $146(13.4)$ & $<0.001$ \\
\hline Myocardial infarction & $142(8.6)$ & $54(9.7)$ & $88(8.1)$ & 0.282 \\
\hline \multicolumn{5}{|l|}{ Personal medical history } \\
\hline Diabetes & $179(10.9)$ & $107(19.2)$ & $72(6.6)$ & $<0.001$ \\
\hline Stroke & $33(2.0)$ & $22(3.9)$ & II (I.0) & $<0.001$ \\
\hline Myocardial infarction & $34(2.1)$ & $20(3.6)$ & $14(1.3)$ & 0.002 \\
\hline Obesity (BMI > 25 kg/m²) & $328(20.0)$ & $156(28.0)$ & $172(15.8)$ & $<0.001$ \\
\hline \multicolumn{5}{|l|}{ Worries about } \\
\hline Hypertension & $887(54.0)$ & 47I (84.4) & $416(38.3)$ & $<0.001$ \\
\hline Diabetes & $775(47.1)$ & $292(52.3)$ & $483(44.5)$ & 0.003 \\
\hline Salt preference & & & & 0.109 \\
\hline Prefers salty foods & $957(58.2)$ & $340(60.9)$ & $617(56.8)$ & \\
\hline Does not prefer salty foods & $687(41.8)$ & $218(39.1)$ & $469(43.2)$ & \\
\hline \multicolumn{5}{|l|}{ Current lifestyle behaviors } \\
\hline Smoking & $258(15.7)$ & $61(10.9)$ & $197(18.1)$ & $<0.001$ \\
\hline Drinking & $663(40.3)$ & $201(36.0)$ & $462(42.5)$ & 0.011 \\
\hline Exercise regularly & $640(38.9)$ & $279(50.0)$ & $361(33.2)$ & $<0.001$ \\
\hline Reducing salt intake & $884(53.8)$ & $406(72.8)$ & $478(44.0)$ & $<0.001$ \\
\hline Behavior stages of reducing salt intake & & & & $<0.001$ \\
\hline Precontemplation & $218(13.3)$ & $43(7.7)$ & $175(16.1)$ & \\
\hline Contemplation & $478(29.1)$ & $95(17.0)$ & $383(35.3)$ & \\
\hline Preparation & $64(3.9)$ & $14(2.5)$ & $50(4.6)$ & \\
\hline Action & $104(6.3)$ & $52(9.3)$ & $52(4.8)$ & \\
\hline Maintain & $780(47.4)$ & $354(63.4)$ & $426(39.2)$ & \\
\hline Willing to be tested & $809(49.2)$ & $313(56.1)$ & $496(45.7)$ & $<0.001$ \\
\hline
\end{tabular}

Note: ${ }^{*}$ Chi-square test.

Abbreviation: BMI, body mass index. 
Table 2 Behavioral changes after notification of test results among participants who had not reduced salt intake

\begin{tabular}{|c|c|c|c|c|}
\hline Behavioral changes & Total, $n=760, n(\%)$ & Hypertension, $n=152, n$ (\%) & Nonhypertension, $\mathrm{n}=608, \mathrm{n}(\%)$ & $P$-value* \\
\hline \multicolumn{5}{|l|}{ If with genetic risk } \\
\hline Behavior stage & & & & 0.001 \\
\hline Precontemplation & $75(9.9)$ & $15(9.9)$ & $60(9.9)$ & \\
\hline Contemplation & $214(28.2)$ & $62(40.8)$ & $152(25.0)$ & \\
\hline Preparation & $35 \mathrm{I}(46.2)$ & $56(36.8)$ & $295(48.5)$ & \\
\hline Action & $120(15.8)$ & $19(12.5)$ & $101(16.6)$ & \\
\hline Behavioral modification & & & & $<0.001$ \\
\hline Progress & $476(62.6)$ & $74(48.7)$ & $402(66.1)$ & \\
\hline No change & $27 \mid(35.7)$ & $75(49.3)$ & $196(32.2)$ & \\
\hline Regress & $13(1.7)$ & $3(2.0)$ & $10(1.6)$ & \\
\hline \multicolumn{5}{|l|}{ If without genetic risk } \\
\hline Behavior stage & & & & 0.692 \\
\hline Precontemplation & $165(2 \mid .7)$ & $33(21.7)$ & $132(2 \mid .7)$ & \\
\hline Contemplation & $396(52.1)$ & $83(54.6)$ & $313(5 \mid .5)$ & \\
\hline Preparation & $170(22.4)$ & $29(19.1)$ & $|4|(23.2)$ & \\
\hline Action & $29(3.8)$ & $7(4.6)$ & $22(3.6)$ & \\
\hline Behavioral modification & & & & 0.126 \\
\hline Progress & $236(31.1)$ & $44(29.0)$ & $192(31.6)$ & \\
\hline No change & $460(60.5)$ & 89 (58.6) & $37 \mid(6 I .0)$ & \\
\hline Regress & $64(8.4)$ & $19(12.5)$ & $45(7.4)$ & \\
\hline
\end{tabular}

Note: *Chi-square test.

Table 3 Behavioral changes after notification of test results among participants who had already reduced salt intake

\begin{tabular}{|c|c|c|c|c|}
\hline Behavioral changes & Total, $n=884, n(\%)$ & Hypertension, $n=406, n$ (\%) & Nonhypertension, $n=478, n(\%)$ & $P$-value* \\
\hline \multicolumn{5}{|l|}{ If with genetic risk } \\
\hline Behavior stage & & & & 0.190 \\
\hline Action & $102(11.5)$ & $52(12.8)$ & $50(10.5)$ & \\
\hline Maintain & $767(86.8)$ & $350(86.2)$ & $417(87.2)$ & \\
\hline Relapse & $15(1.7)$ & $4(1.0)$ & II (2.3) & \\
\hline Behavioral modification & & & & 0.131 \\
\hline No change & $69(98.3)$ & $402(99.0)$ & $467(97.7)$ & \\
\hline Relapse & $15(1.7)$ & $4(1.0)$ & II (2.3) & \\
\hline \multicolumn{5}{|l|}{ If without genetic risk } \\
\hline Behavior stage & & & & 0.267 \\
\hline Action & $97(11.0)$ & $48(11.8)$ & $49(10.3)$ & \\
\hline Maintain & $708(80.1)$ & $316(77.8)$ & $392(82.0)$ & \\
\hline Relapse & $79(8.9)$ & $42(10.3)$ & $37(7.7)$ & \\
\hline Behavioral modification & & & & 0.176 \\
\hline No change & $805(91.1)$ & $364(89.7)$ & $44 \mid(92.3)$ & \\
\hline Relapse & $79(8.9)$ & $42(10.3)$ & $37(7.7)$ & \\
\hline
\end{tabular}

Note: *Chi-square test.

between the two groups was detected after being notified of the result "If with genetic risk" or "If without genetic risk" (Table 3).

Compared to hypertensive patients, nonhypertensive patients were more likely to make positive behavioral modifications after being notified of the result "If with genetic risk" (hypertensive patients vs nonhypertensive patients, $48.7 \%$ vs $66.1 \% ; P<0.001$, chi-square test, Figure 1). On the other hand, compared to nonhypertensive patients, hypertensive patients were more likely to make negative behavioral modifications after being notified of the result "If without genetic risk" (10.9\% vs $7.6 \% ; P=0.021)$.
Univariate logistic regression analysis (Table 4) revealed that in all participants and those aged $<65$ years, compared to hypertensive patients, nonhypertensive patients were more likely to make positive behavioral modifications after being notified of the result "If with genetic risk" ([crude RR $\{c-R R\}, 2.06 ; 95 \% \mathrm{CI}, 1.44-2.95]$ and [c-RR, 2.24; $95 \% \mathrm{CI}$, 1.37-3.67], respectively). In contrast, in all patients, compared to the hypertensive patients, nonhypertensive patients were unlikely to make negative behavioral modifications after being notified of the result "If without genetic risk" (c-RR, $0.67 ; 95 \%$ CI, 0.47-0.94). Furthermore, multivariate logistic regression analysis identified that in all participants and those 


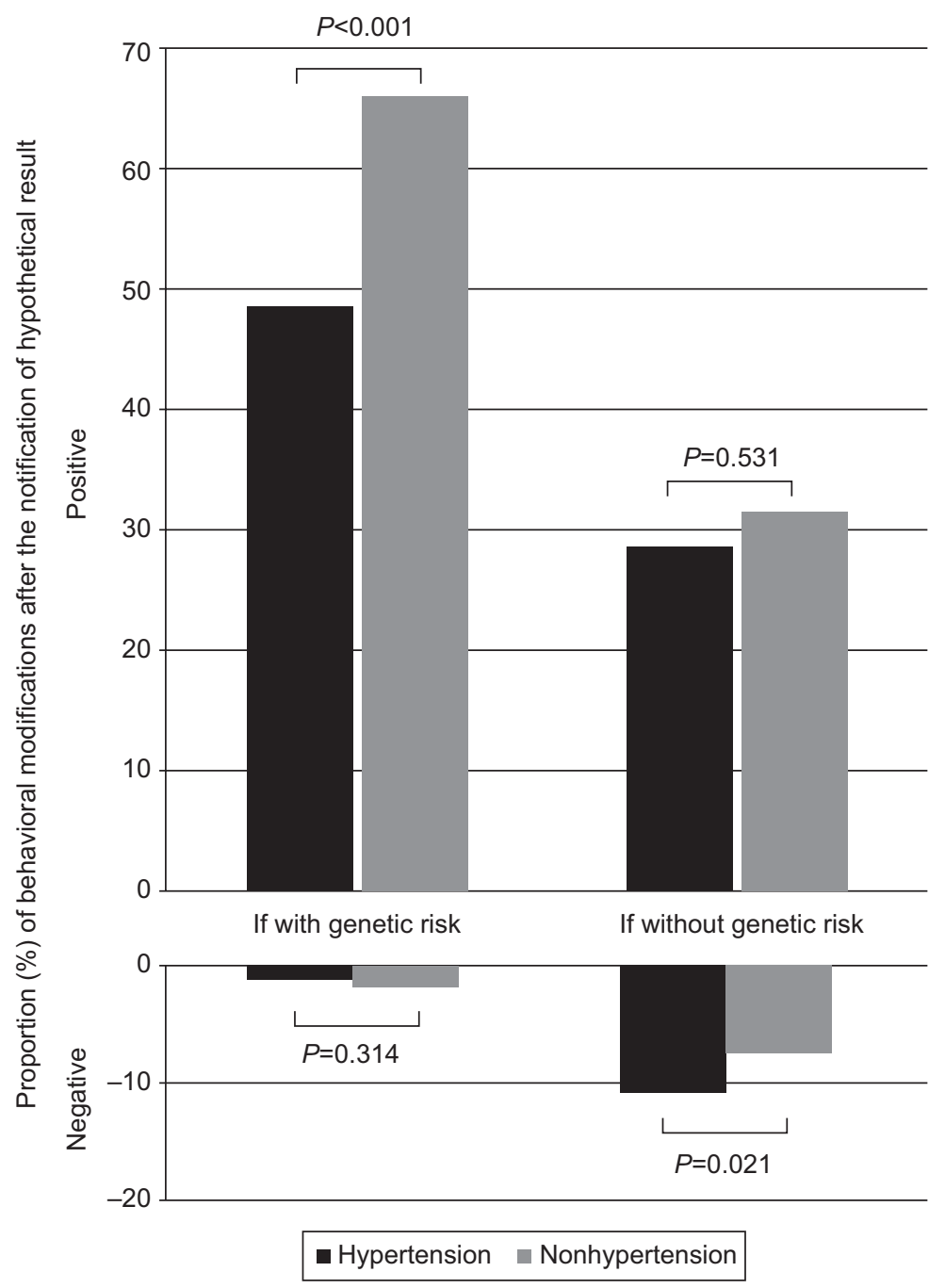

Figure I Behavioral modifications following notification of hypothetical genetic test results of salt sensitivity.

Notes: The proportions of positive and negative behavioral modifications as a result of notification of hypothetical results in the hypertensive patients (black) and nonhypertensive patient (light gray) groups are presented. The proportion of positive behavioral modifications in the hypertensive patients ( $\mathrm{n}=\mathrm{I52}$ ) and nonhypertensive patients $(n=608)$ were $48.7 \%(n=74)$ and $66.1 \%(n=402)$, respectively, after being notified of the result "If with genetic risk" $(P<0.001$, chi-square test); and $29.0 \%(n=44)$ and $31.6 \%(n=192)$, respectively, after being notified of the result "If without genetic risk" ( $P=0.531$, chi-square test). The proportion of negative behavioral modifications in hypertensive patients $(n=558)$ and nonhypertensive patients $(n=I, 086)$ were $I .3 \%(n=7)$ and I.9\% $(n=2 I)$, respectively, after being notified of the result "If with genetic risk" $(P=0.314$, chi-square test) and $10.9 \%(n=6 I)$ and $7.6 \%(n=82)$, respectively, after being notified of the result "If without genetic risk" ( $P=0.021$, chi-square test).

Table 4 Relative ratios of behavioral modifications after notifications of genetic risk between hypertensive and nonhypertensive patients

\begin{tabular}{|c|c|c|c|c|c|c|c|c|c|}
\hline \multirow{2}{*}{$\begin{array}{l}\text { Behavioral } \\
\text { modification }\end{array}$} & \multicolumn{3}{|c|}{$<65$ years } & \multicolumn{3}{|c|}{$\geq 65$ years } & \multicolumn{3}{|l|}{ Total } \\
\hline & $\mathbf{n}$ & $\begin{array}{l}\text { c-RR,* } \\
95 \% \mathrm{Cl}\end{array}$ & $\begin{array}{l}\text { ad-RR, }{ }^{* \#} \\
95 \% \mathrm{CI}\end{array}$ & $\mathbf{n}$ & $\begin{array}{l}\text { c-RR,* } \\
95 \% \mathrm{Cl}\end{array}$ & $\begin{array}{l}\text { ad-RR, }{ }^{* \#} \\
95 \% \mathrm{Cl}\end{array}$ & $\mathbf{n}$ & $\begin{array}{l}\text { c-RR, } \\
95 \% \mathrm{Cl}\end{array}$ & $\begin{array}{l}\text { ad-RR, } \\
95 \% \text { Cl }\end{array}$ \\
\hline \multicolumn{10}{|l|}{ If with genetic risk } \\
\hline Positive effect & 601 & $2.24,1.37-3.67$ & $1.99,1.11-3.57$ & 159 & $1.08,0.58-2.01$ & $1.60,0.76-3.39$ & 760 & $2.06,1.44-2.95$ & $1.76,1.12-2.76$ \\
\hline Negative effect & $\mathrm{I}, 005$ & $1.99,0.46-8.63$ & $2.60,0.54-12.4$ & 639 & $0.87,0.21-3.66$ & $0.70,0.16-3.15$ & $\mathrm{I}, 644$ & $1.55,0.66-3.67$ & $1.40,0.52-3.75$ \\
\hline \multicolumn{10}{|c|}{ If without genetic risk } \\
\hline Positive effect & 601 & I.3I, 0.76-2.27 & $1.27,0.69-2.35$ & 159 & $0.62,0.30-1.27$ & $0.62,0.27-1.42$ & 760 & $1.13,0.77-1.67$ & $0.97,0.6 \mathrm{I}-\mathrm{I} .55$ \\
\hline Negative effect & $\mathrm{I}, 005$ & $0.63,0.35-1.14$ & $0.68,0.35-1.32$ & 639 & I.II, 0.69-1.78 & $1.26,0.76-2.08$ & $\mathrm{I}, 644$ & $0.67,0.47-0.94$ & $1.05,0.70-1.57$ \\
\hline
\end{tabular}

Notes: *RR of the nonhypertension group with reference to the hypertension group; \#adjusted for age ( $<65$ vs $\geq 65$ years), sex (male vs female), education level (high school or lower vs college and university), preferring salty foods (yes vs no), behavior stages before being notified of the genetic results (contemplation, preparation, action, and maintain vs precontemplation), and willingness to be tested (yes vs no).

Abbreviations: $\mathrm{c}-\mathrm{RR}$, crude relative ratio; $\mathrm{Cl}$, confidence interval; ad-RR, adjusted relative ratio. 
aged $<65$ years, compared to the hypertensive patients, nonhypertensive patients were significantly more likely to make positive behavioral modification after being notified of the result "If with genetic risk" ([adjusted RR \{ad-RR\}, 1.76; 95\% CI, 1.12-2.76] and [ad-RR, 1.99; 95\% CI, 1.11-3.57], respectively). No significant difference was detected in negative behavioral modification after being notified of the result "If without genetic risk" between hypertensive and nonhypertensive patients (ad-RR, 1.05; 95\% CI, 0.70-1.57; Table 4).

\section{Discussion}

Previous studies have found that, in comparison with healthy controls, patients with breast cancer were significantly more interested in undergoing genetic testing ${ }^{27}$ and that the majority of ovarian cancer patients were willing to be tested, if the genetic testing would benefit their family in terms of prediction of disease ${ }^{28}$ Similarly, the findings of our study indicated that a medical history of hypertension could influence the effect of disclosing the genetic risk on patients behavioral intention and that the behavioral modification after being notified of hypothetical genetic testing results for salt-sensitive hypertension differed between hypertensive and nonhypertensive patients. The lack of assessment of patients' medical histories in previous studies on the topic could help explain the mixed findings of these reports, and hence, we propose that assessing the medical history of study participants is necessary to design trials exploring the effects of disclosing the genetic risk.

We found that about twice as many nonhypertensive patients as hypertensive patients made progress in their behavior of reducing salt intake after being notified of the result "If with the genetic risk". In the process of the behavioral change, feelings concerning importance, such as personal values and expectations of the importance of change, and confidence have been shown to contribute to the general state of readiness to change. ${ }^{29}$ The role of outcome expectancy is essential in designing effective behavioral interventions, ${ }^{30}$ with the outcome expectancy of nonhypertensive patients for reducing salt intake generally being the prevention of hypertension. The findings of this study indicate that disclosing the genetic risk for salt sensitivity could bring positive behavioral modifications with regard to salt intake.

Nutrigenetics aims to understand how the genetic makeup of an individual coordinates the response to $\operatorname{diet}^{31}$ and is a promising tool for improving our knowledge about the nutrition required for optimal personal health. ${ }^{32}$ High salt intake is associated with high blood pressure, and salt-sensitive normotensive individuals have a higher rate of incident hypertension. ${ }^{33,34}$ A modest reduction in salt intake for 4 or more weeks has been demonstrated to result in decreased blood pressure in both hypertensive and normotensive individuals. ${ }^{35}$ Behavioral modification is more efficient for the prevention of hypertension in young adults and adults aged $<65$ years, compared to adults aged $\geq 65$ years. In this study, after being notified of the result "If with genetic risk", nonhypertensive patients aged $<65$ years were more likely to make positive behavioral modifications compared to hypertensive patients. As a result of positive behavioral modifications with regard to salt intake, the disclosure of the genetic risk for salt-sensitive hypertension could help prevent the development of hypertension.

Furthermore, no difference in negative behavioral modifications between hypertensive and nonhypertensive patients was detected in this study after being notified of the result "If without genetic risk", indicating that a medical history of hypertension would not influence the effect of the notification of "no genetic risk for salt sensitivity" on the behavioral changes of these patients. However, in our previous study, after disclosing negative test results, the patients who had already reduced salt intake, especially those with a salt preference, were likely to quit the salt restriction. ${ }^{13}$

High salt intake is associated with increased rates of cardiovascular disease, ${ }^{33}$ and lower sodium intake is associated with a reduced risk of strokes and fatal coronary heart disease in adults. ${ }^{36}$ Hence, although the limits of salt intake in terms of safety are unclear, recommendations for reducing the current levels of salt consumption seem justifiable. ${ }^{33}$ All hypertensive patients have salt-sensitive traits, and among hypertensive patients, $51 \%$ are thought to have salt-sensitive blood pressure. ${ }^{37}$ The present study showed that $10.9 \%$ of hypertensive patients showed negative behavioral changes with regard to reducing salt intake. Hence, the disclosure of negative results may lead hypertensive patients to reduce the preventative measures taken to control their blood pressure. Counseling may be important after genetic testing, particularly when a negative result is provided.

This study has several limitations. First, the findings in this study are based on the hypothetical genetic testing: "a genetic testing that detects whether or not you have a genetic risk predisposing you to hypertension by excessive salt intake". The impact of the hypothetical genetic testing results probably did not differ from the impact produced by actual salt-sensitivity genetic test results, but further trials are required to measure behavioral modifications in response to actual salt-sensitivity genetic test results. In addition, more genetic information concerning salt-sensitive 
genetic testing would typically be presented in real life than in this study. The influence of genetic information on the behavior of salt intake might be different in each setting, but further trials are required to examine this issue. Second, we compared the difference in behavioral modifications between hypertensive and nonhypertensive patients after being notified of the genetic risk. In each hypertensive and nonhypertensive group, comparison between patients who were informed of their genetic status and those who were not and between those who were informed of salt-sensitivity hypertension-related genetic tests and those who were not would be more valid. Third, low salt campaigns by local governments, nonprofit organizations, and mass media can be seen all over Japan, ${ }^{38}$ and although the education statuses of the patients were measured in this study, their knowledge concerning the relationship between salt intake and hypertension was not questioned, and this might further influence the degree of behavioral modification. Fourth, we did not measure the grade of the participants' worries about hypertension. This might have an influence on behavioral modification; however, the degree of influence was unknown. Fifth, we did not measure the actual daily salt intake; it is reported that some single polymorphisms may modify salt taste perception, ${ }^{39}$ although patient preference for salty foods does not always correlate with salt intake. ${ }^{40}$ Future studies should explore the relationship between actual daily salt intake and these SNPs, while disclosing the genetic risk for salt-sensitive hypertension. Sixth, the definition of hypertensive patients was not based on their blood pressure. The anonymous questionnaires were distributed among the participants in the waiting room and they filled out the questionnaire at the same place; the clinical data were not obtained in this study. Therefore, the decision regarding whether the participants were hypertensives or nonhypertensives was made on the basis of their personal medical record in this study. Seventh, the ratio of male to female patients visiting the six primary care clinics and hospitals was not obtained in this study. It is unclear how representative the sample is of the practice populations. Eighth, all the participants in this study were Japanese in origin. Cultural, dietary, and genetic differences might have diminished or reinforced the applicability of the findings in this study, especially, differences in the daily salt intake might influence the interpretation of the findings in this study. Finally, we did not evaluate the effects of disclosure of a hypothetical genetic test result in terms of quality of life, control of hypertension, and prevention of an adverse cardiovascular outcome. In addition, we did not perform a qualitative approach concerning the mechanism of motivation for the lower salt intake. Future studies are required to clarify these issues.

\section{Conclusion}

The behavior of modifying salt intake after disclosure of genetic testing results differed between hypertensive and nonhypertensive patients, and we conclude that assessment of patients' medical histories would be necessary to design studies exploring the effects of disclosing the genetic risk. In our study, we found that disclosing a genetic risk for saltsensitive hypertension was likely to cause nonhypertensive patients, especially those aged $<65$ years, to improve their behavior regarding salt intake and general health. Hence, we hypothesize that the disclosure of genetic testing results could help prevent hypertension by bringing a change in the behavioral modification with respect to salt intake. In contrast, after being notified of the negative results for salt-sensitivity, hypertensive patients did not differ in making negative behavioral changes from nonhypertensive patients. However, because of the possibility of negative behavioral changes of reducing salt intake, disclosing the negative results may lead to reduced control of the blood pressure in hypertensive patients. Although further studies are needed to determine the effects of disclosing genetic testing results, we conclude that different genetic counseling strategies should be provided based on the patients' medical histories.

\section{Acknowledgments}

The authors thank S Fujiwara, MD, PhD, D Matsuhima, MD, $\mathrm{PhD}, \mathrm{K}$ Nakayasu, MD, C Ishida, MD, Y Ishibashi, MD, S Ohguri, R Kobayashi, and K Kawabata for their assistance with data collection. This study was supported by a Grant-inAid for Scientific Research (B) (21390168) from the Japan Society for the Promotion of Science.

This paper was presented at the WONCA Asia Pacific Regional Conference 2014 as a poster presentation with interim findings. The poster's abstract was published in "Poster Abstracts" in Malaysian Family Physician, Official Journal of the Academy of Family Physicians of Malaysia, and Family Medicine Specialists Association, WONCA Asia Pacific Regional Conference 2014.

\section{Author contributions}

MO and TT contributed to the conception, design, and acquisition of data; the analysis and interpretation of data; and the writing and revision of the manuscript. MH and RA 
contributed to the design and acquisition of data, the analysis and interpretation of data, and the writing and revision of the manuscript. EK contributed to the conception and design and the writing and revision of the manuscript.

\section{Disclosure}

The authors report no conflicts of interest in this work.

\section{References}

1. Staessen JA, Wang J, Bianchi G, Birkenhager WH. Essential hypertension. Lancet. 2003;361(9369):1629-1641.

2. Basile J, Bloch MJ. Overview of hypertension in adults. UpToDate [updated March 2, 2016]. Available from: http://www.uptodate.com/ contents/overview-of-hypertension-in-adults?source=search_result\&s earch=hypertension\&selectedTitle=1 150. Accessed April 21, 2016.

3. Kupper N, Willemsen G, Riese H, Posthuma D, Boomsma DI, de Geus EJ. Heritability of daytime ambulatory blood pressure in an extended twin design. Hypertension. 2005;45(1):80-85.

4. A catalog of published genome-wide association studies [homepage on the Internet] [updated September 16, 2015]. Available from: https:// www.genome.gov/GWAStudies/. Accessed April 21, 2016.

5. Zeller T, Blankenberg S, Diemert P. Genomewide association studies in cardiovascular disease - an update 2011. Clin Chem. 2012;58(1):92-103.

6. Tuckson RV, Newcomer L, De Sa JM. Accessing genomic medicine: affordability, diffusion, and disparities. JAMA. 2013;309(14): 1469-1470.

7. Dube JB, Hegele RA. Genetics 100 for cardiologists: basics of genomewide association studies. Can J Cardiol. 2013;29(1):10-17.

8. Khoury MJ, McBride CM, Schully SD, et al. The Scientific Foundation for personal genomics: recommendations from a National Institutes of Health-Centers for Disease Control and Prevention multidisciplinary workshop. Genet Med. 2009;11(8):559-567.

9. Scheuner MT, Sieverding P, Shekelle PG. Delivery of genomic medicine for common chronic adult diseases: a systematic review. JAMA. 2008;299(11):1320-1334.

10. Bloss CS, Schork NJ, Topol EJ. Effect of direct-to-consumer genomewide profiling to assess disease risk. $N$ Engl J Med. 2011;364(6):524-534.

11. James KM, Cowl CT, Tilburt JC, et al. Impact of direct-to-consumer predictive genomic testing on risk perception and worry among patients receiving routine care in a preventive health clinic. Mayo Clin Proc. 2011;86(10):933-940.

12. EGAPP Working Group. Recommendations from the EGAPP Working Group: does genomic profiling to assess type 2 diabetes risk improve health outcomes? Genet Med. 2013;15(8):612-617.

13. Takeshima T, Okayama M, Harada M, Ae R, Kajii E. Effects of disclosing hypothetical genetic test results for salt sensitivity on salt restriction behavior. Int J Gen Med. 2013;6:361-368.

14. Vassy JL, Donelan K, Hivert MF, Green RC, Grant RW. Genetic susceptibility testing for chronic disease and intention for behavior change in healthy young adults. J Community Genet. 2013;4(2):263-271.

15. Marsaux CF, Celis-Morales C, Livingstone KM, et al. Changes in physical activity following a genetic-based Internet-delivered personalized intervention: randomized controlled trial (food $4 \mathrm{me}) . J$ Med Internet Res. 2016;18(2): $\mathrm{e} 30$

16. Christensen KD, Roberts JS, Whitehouse PJ, et al. Disclosing pleiotropic effects during genetic risk assessment for Alzheimer disease: a randomized trial. Ann Intern Med. 2016;164(3):155-163.

17. Nielsen DE, El-Sohemy A. Disclosure of genetic information and change in dietary intake: a randomized controlled trial. PLoS One. 2014;9(11):e112665.

18. Hutson SP. Attitudes and psychological impact of genetic testing, genetic counseling, and breast cancer risk assessment among women at increased risk. Oncol Nurs Forum. 2003;30(2):241-246.
19. Taylor RS, Ashton KE, Moxham T, Hooper L, Ebrahim S. Reduced dietary salt for the prevention of cardiovascular disease: a meta-analysis of randomized controlled trials (Cochrane review). Am J Hypertens. 2011;24(8):843-853.

20. Yang Q, Liu T, Kuklina EV, et al. Sodium and potassium intake and mortality among US adults: prospective data from the Third National Health and Nutrition Examination Survey. Arch Intern Med. 2011;171(13): 1183-1191.

21. Zhao Q, Gu D, Hixson JE, et al. Common variants in epithelial sodium channel genes contribute to salt sensitivity of blood pressure: The GenSalt study. Circ Cardiovasc Genet. 2011;4(4):375-380.

22. Sanada H, Yatabe J, Midorikawa S, et al. Single-nucleotide polymorphisms for diagnosis of salt-sensitive hypertension. Clin Chem. 2006;52(3):352-360.

23. Katsuya T, Ishikawa K, Sugimoto K, Rakugi H, Ogihara T. Salt sensitivity of Japanese from the viewpoint of gene polymorphism. Hypertens Res. 2003;26(7):521-525.

24. Exploratory Committee on Standard Methods for Health-Check and Health Guidance. A questionnaire for evaluating behavioral stages of lifestyle [updated March 26, 2007]. Available from: http://www.mhlw. go.jp/bunya/kenkou/seikatsu/pdf/03-c-06.pdf. Accessed April 21, 2016.

25. Kanazawa M, Yoshiike N, Osaka T, Numba Y, Zimmet P, Inoue S. Criteria and classification of obesity in Japan and Asia-Oceania. World Rev Nutr Diet. 2005;94:1-12.

26. Weinstein ND, Rothman AJ, Sutton SR. Stage theories of health behavior: conceptual and methodological issues. Health Psychol. 1998;17(3):290-299.

27. Bruno M, Digennaro M, Tommasi S, et al. Attitude towards genetic testing for breast cancer susceptibility: a comparison of affected and unaffected women. Eur J Cancer Care (Engl). 2010;19(3):360-368.

28. Lacour RA, Daniels MS, Westin SN, et al. What women with ovarian cancer think and know about genetic testing. Gynecol Oncol. 2008;111(1):132-136.

29. Rollnick S, Mason P, Butler C. Health Behavior Change. Edinburgh: Churchill Livingstone; 1999.

30. Williams DM, Anderson ES, Winett RA. A review of the outcome expectancy construct in physical activity research. Ann Behav Med. 2005;29(1):70-79.

31. Mutch DM, Wahli W, Williamson G. Nutrigenomics and nutrigenetics: the emerging faces of nutrition. FASEB J. 2005;19(12):1602-1616.

32. Phillips CM. Nutrigenetics and metabolic disease: current status and implications for personalised nutrition. Nutrients. 2013;5(1):32-57.

33. Kotchen TA, Cowley AW, Jr, Frohlich ED. Salt in health and disease - a delicate balance. N Engl J Med. 2013;368(26):2531-2532.

34. Barba G, Galletti F, Cappuccio FP, et al. Incidence of hypertension in individuals with different blood pressure salt-sensitivity: results of a 15-year follow-up study. J Hypertens. 2007;25(7):1465-1471.

35. He FJ, Li J, Macgregor GA. Effect of longer term modest salt reduction on blood pressure: Cochrane systematic review and meta-analysis of randomised trials. BMJ. 2013;346:f1325.

36. Aburto NJ, Ziolkovska A, Hooper L, Elliott P, Cappuccio FP, Meerpohl JJ. Effect of lower sodium intake on health: systematic review and metaanalyses. BMJ. 2013;346:f1326.

37. Weinberger MH, Miller JZ, Luft FC, Grim CE, Fineberg NS. Definitions and characteristics of sodium sensitivity and blood pressure resistance. Hypertension. 1986;8(6 Pt 2):II127-134.

38. Ministry of Health Labor and Welfare. Diet and hypertension. e-Eealthnet, Health information. Available from: http://gen-en.net/news.html. Accessed April 21, 2016.

39. Dias AG, Rousseau D, Duizer L, et al. Genetic variation in putative salt taste receptors and salt taste perception in humans. Chem Senses. 2013;38(2):137-145.

40. Hashimoto T, Yagami F, Owada M, Sugawara T, Kawamura M. Salt preference according to a questionnaire vs. dietary salt intake estimated by a spot urine method in participants at a health check-up center. Intern Med. 2008;47(5):399-403. 


\section{Publish your work in this journal}

The International Journal of General Medicine is an international, peer-reviewed open-access journal that focuses on general and internal medicine, pathogenesis, epidemiology, diagnosis, monitoring and treatment protocols. The journal is characterized by the rapid reporting of reviews, original research and clinical studies across all disease areas.
The manuscript management system is completely online and includes a very quick and fair peer-review system, which is all easy to use. Visit http://www.dovepress.com/testimonials.php to read real quotes from published authors.

Submit your manuscript here: https://www.dovepress.com/international-journal-of-general-medicine-journal 